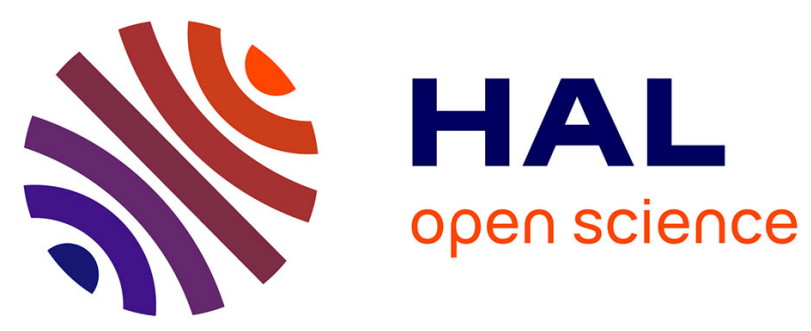

\title{
Health-related quality of life of patients with pulmonary arterial hypertension associated with CHD: the multicentre cross-sectional ACHILLE study
}

Pascal Amedro, Adeline Basquin, Virginie Gressin, Pierre Clerson, Xavier

Jaïs, Jean-Benoit Thambo, Patrice Guerin, Sarah Cohen, Damien Bonnet

\section{To cite this version:}

Pascal Amedro, Adeline Basquin, Virginie Gressin, Pierre Clerson, Xavier Jaïs, et al.. Health-related quality of life of patients with pulmonary arterial hypertension associated with CHD: the multicentre cross-sectional ACHILLE study. Cardiology in the Young, 2016, 26 (07), pp.1250 - 1259. 10.1017/S1047951116000056 . hal-01792538

\section{HAL Id: hal-01792538 \\ https://hal.umontpellier.fr/hal-01792538}

Submitted on 23 Nov 2019

HAL is a multi-disciplinary open access archive for the deposit and dissemination of scientific research documents, whether they are published or not. The documents may come from teaching and research institutions in France or abroad, or from public or private research centers.
L'archive ouverte pluridisciplinaire HAL, est destinée au dépôt et à la diffusion de documents scientifiques de niveau recherche, publiés ou non, émanant des établissements d'enseignement et de recherche français ou étrangers, des laboratoires publics ou privés. 


\section{Health-related quality of life of patients with pulmonary arterial hypertension associated with CHD: the multicenter cross-sectional ACHILLE study}

Pascal Amedro, 1,2 Adeline Basquin,3 Virginie Gressin,4 Pierre Clerson, 5 Xavier Jais, 6 Jean-Benoit Thambo,7Patrice Guerin,8 Sarah Cohen,9,10 Damien Bonnet 11

1Pediatric and Congenital Cardiology, Arnaud de Villeneuve University Hospital, PhyMedexp, INSERM U1046,CNRS UMR 9214, University of Montpellier, Montpellier; 2Department of Public Health, EA3279 Self-perceived Health Assessment Research Unit, Aix-Marseille University, Marseille;

3Pediatric and Congenital Cardiology, UniversityHospital, Rennes;

4Actelion Pharmaceuticals France, Paris;

${ }_{5}$ Orgametrie Biostatistics, Roubaix;

6Bicêtre University Hospital, Le Kremlin-Bicêtre;

7Pediatric and Congenital Cardiology, Haut-Leveque University Hospital, Bordeaux;

8Pediatric and Congenital Cardiology, Guillaume and René Laennec University Hospital, Nantes;

9Adult CongenitalHeart Disease Unit, M3C-George Pompidou European Hospital, APHP, Paris Descartes University;

10INSERMUMRS 1138, Cordelier Research Center, Team 22;

${ }_{11}$ Pediatric and Congenital Cardiology, M3C-Necker University Hospital, AP-HP, Paris

Descartes University, Paris, France

Background: The aim of this study was to assess health-related quality of life in patients with pulmonary arterial hypertension associated with $\mathrm{CHD}$ and correlations with clinical status.

Methods: This prospective cross-sectional observational study included CHD patients with pulmonary arterial hypertension in 14 tertiary-care centres in France. We used two health-related quality of life questionnaires - SF-36 and Cambridge Pulmonary Hypertension Outcome Review (CAMPHOR) - and one anxiety/depression Hospital Anxiety and Depression Scale (HADS) questionnaire.

Results: Clinical data were collected for the 208 included patients (mean age: 42.6 years, range from 15.1 to 85.8 years, $69.7 \%$ female). Most patients were in NYHA functional class II (48.1\%) and III (37.5\%). Patients' phenotype was classified as Eisenmenger syndrome $(70.7 \%)$, pulmonary arterial hypertension associated with systemic-to-pulmonary shunts $(12.0 \%)$, with small defects $(3.4 \%)$, or after corrective cardiac surgery (13.9\%). In total, $76.4 \%$ of the patients were receiving pulmonary arterial hypertension-specific treatments. SF-36 scores showed impairment compared with normalised data. Health-related quality of life scores were significantly lower in females than in males for most dimensions of both questionnaires and were independent of the patients' phenotype, even after gender adjustment - except for CAMPHOR functioning but significantly depended on NYHA functional class. The Hospital Anxiety and Depression Scale (HADS) scores suggested anxiety and depression associated with increasing NYHA functional class but independent of patients' phenotype. NYHA functional class, 6-minute walk distance, HADS, gender, and recent stressful event significantly affected quality of life in the multivariate analysis.

Conclusions: This study showed impairment of quality of life in a large cohort of patients with pulmonary arterial hypertension associated with CHD with both generic and specific questionnaires. NYHA functional class and HADS scores were predictive of most quality of life scores. 
Keywords: Health-related quality of life; pulmonary arterial hypertension; CHD; Eisenmenger syndrome; CAMPHOR

CHD are the most common of all congenital malformations with an estimated incidence of $8 / 1000$ births. 1 As a consequence of paediatric medical advances, prevalence of adults with CHD has been progressively increasing throughout the past two decades.2 The median age at death for complex CHD has switched from childhood to adulthood and, for instance, increased from 2 to 23 years in the Canadian registry.3 Pulmonary arterial hypertension is a severe and frequent (5-10\%) complication of $\mathrm{CHD}_{4}$ involving vascular re-modelling, increased pulmonary vascular resistance, and elevated pulmonary arterial pressure.5 In CHD patients, a diagnosis of pulmonary arterial hypertension multiplies by two the risk of mortality, by three the risk of cardiac event, and by five the risk of hospitalisation in ICUs.6 At present, patients developing pulmonary arterial hypertension associated with CHD are managed in tertiary-care centres, where the introduction of advanced specific therapies is expected to improve survival.7 In this new epidemiologic context, the quality of life of CHD patients with pulmonary arterial hypertension deserves investigation. Indeed, these patients suffer from altered physical abilities and must bear the emotional and psychological consequences of a progressive disease.8,9 Health-related quality of life evaluation has become an emerging research theme in congenital cardiology and an important end point among patientreported outcomes in pulmonary hypertension clinical studies;10,11 however, limited health-related quality of life data are available for CHD patients with pulmonary arterial hypertension.1217 The ACHILLE - Adults adolescents CHD with pulmonary arterial hypertension quality of life assessment - study aimed to evaluate health-related quality of life in a large population of CHD patients with pulmonary arterial hypertension using a generic (SF-36) and a pulmonary hypertension-specific (Cambridge Pulmonary Hypertension Outcome Review (CAMPHOR)) instrument.

\section{Materials and methods}

Study design This prospective, cross-sectional, observational study was carried out in 14 tertiary-care CHD centres in France, and enrolled patients from May, 2012 to June, 2013.

The study was conducted in compliance with the Good Clinical Practices protocol and Declaration of Helsinki principles. In accordance with French law, formal approval from an ethical committee was not required for this observational study. Patients or their guardians gave their oral consent.

Population Patients over 15 years of age with pulmonary arterial hypertension associated with CHD were asked to participate in the study during routine follow-up. Pulmonary arterial hypertension was confirmed by right heart catheterisation, and pulmonary arterial hypertension associated with CHD was classified according to the Dana Point clinical classification 18 as Eisenmenger syndrome, pulmonary arterial hypertension associated with systemic-to-pulmonary shunts, pulmonary arterial hypertension associated with small defects, and pulmonary arterial hypertension after corrective cardiac surgery. Patients with typical Eisenmenger syndrome - long-standing left-to-right shunt reversed into a right-to-left shunt - could be enrolled on the basis of the echocardiography. Patients were not eligible if they had undergone heart transplantation, cardiac surgery without subsequent control, planned corrective heart surgery, and recent surgical or interventional catheter procedures ( $<6$ months). Patients who were not able to fill out the questionnaires were excluded. Patients with Down syndrome or any other 
mental disorder were eligible if, upon the investigator's statement, they were able to understand and fill out the questionnaires by themselves.

Data collected Demographic data were recorded. Clinical data included co-morbidities, hospitalisations due to pulmonary arterial hypertension and/or CHD within the last year, stability of the disease status as assessed by the investigator, recent stressful event as reported by the patient, NYHA functional class at inclusion as assessed by the investigator, most recent 6-minute walk distance, and on-going pulmonary arterial hypertension-specific treatments.

Patient reported outcomes In this study we used two health-related quality of life and one anxiety/depression self-reported questionnaires: the Medical Outcome Study 36-item Short Form Health Survey (SF-36v2, Acute, France), the CAMPHOR, and the HADS. The SF-36 is a generic health-related quality of life questionnaire.19 In addition to change in reported health, the domains assessed are physical functioning, role physical, bodily pain, general health, vitality, social functioning, role emotional, and mental health. These domains are combined to formtwo higher-order summaries: the physical and mental health component summaries. Each domain and each summary is reported on a 0-100 scale. Higher scores indicate better health-related quality of life. SF-36 scores are normalised to a mean of 50 and an SD of 10 based on the normal United States population.20 The SF-36 has received linguistic and psychometric validation in French,21,22 and standard (United States-derived) scoring algorithms are recommended for use in France.20 The CAMPHOR is a pulmonary hypertensionspecific health-related quality of life questionnaire.23 It includes three scales designed to assess symptoms, functioning, and quality of life. Scores range from 0 to 25 for the symptom and quality of life scales and from 0 to 30 for the functioning scale. Higher scores indicate greater impairment. The French-Canadian validated version of the CAMPHOR 24 is used in the present study, with permission. The HADS questionnaire 25 is divided into two scales including seven items each for the screening of depression and anxiety. The total scores for depression and for anxiety range between 0 and 21 . Higher scores indicate greater depression and/or anxiety. We considered a score $\geqslant 8$ to represent symptoms of anxiety or depression. 26

Statistical analysis Continuous variables are described as mean \pm standard deviation. Categorical data are described as numbers and percentages for each modality. Characteristics of patients were compared according to the type of pulmonary arterial hypertension associated with CHD or NYHA functional class by analysis of variance for continuous variables and $\chi_{2}$ or exact Fisher's test for categorical variables. Pearson's correlation coefficients were estimated between SF-36 dimensions and CAMPHOR dimensions. Univariate and multivariate regression analyses were performed to identify the covariates impacting the health-related quality of life among the following: gender, age per quartile, time since diagnosis of pulmonary arterial hypertension, depression and anxiety scores, 6-minute walk distance, NYHA functional class, at least one hospitalisation due to pulmonary arterial hypertension and/or CHD in the past 12 months, and recent stressful event. All the variables were introduced in the multivariate model without selection. For all statistical tests, a significance level of $p \leqslant 0.05$ was used.

\section{Results}

Population characteristics 
The characteristics of the 208 evaluated patients are presented in Table 1. Most patients had Eisenmenger syndrome (70.7\%). At inclusion, most patients $(86.0 \%)$ were considered as stable for the previous 3 months, $48.1 \%$ were in NYHA functional class II, $37.5 \%$ in class $\mathrm{III}$, and $76.4 \%$ were receiving pulmonary arterial hypertension-specific treatments. Health-related quality of life scores Mean SF-36 scores were low compared with the normalized mean of 50 for all domains (Fig 1) and also for the summaries indicating decreased health-related quality of life compared with normalised data. When patients were asked to appreciate their health at the time of the study, compared with their health a year before, $59.1 \%$ considered their health as stable, $22.1 \%$ as better, and $18.8 \%$ as worse. SF-36 scores were lower for female than for male patients $(p<0.05$, except for physical component summary: $p=0.053$, role physical: $p=0.07$, and vitality: $p=0.16$ ), but did not vary significantly with the phenotype of pulmonary arterial hypertension associated with CHD (Table 2), even after gender adjustment. All SF-36 scores - individual domains and summaries - were significantly different between NYHA functional class, with and without gender adjustment (Fig 2).

Table 1. Patient characteristics at inclusion.

Patients with

PAH-CHD $(n=208)$

Demographics

Age, years (range)

Female gender (n (\%))

$42.6 \pm 15.8(15.1 ; 85.8)$

Clinical characteristics

$145(69.7)$

Age at CHD diagnosis (n (\%))

Fetal

$<1$ month

$5(2.4)$

1 month to 1 year

$41(19.8)$

$36(17.4)$

$125(60.4)$

$\geqslant 1$ year

Unknown

Age at $\mathrm{PAH}$ diagnosis (years (range))

1

$\mathrm{PAH}-\mathrm{CHD}$ classification ( $\mathrm{n}(\%)$ )

Eisenmenger syndrome

$25.9 \pm 20.3(0 ; 80.0)$

PAH with systemic-pulmonary shunt

$147(70.7)$

$\mathrm{PAH}$ with small defects

$25(12.0)$

7 (3.4)

$\mathrm{PAH}$ after corrective cardiac surgery

$29(13.9)$

Co-morbidities (n (\%))

Respiratory disorder

$20(9.6)$

Neurological disorder

$10(4.8)$

Renal disorder

$7(3.4)$

Liver disorder

$1(0.5)$

Other disorder

$31(14.9)$

Recent stressful event as reported by the

patient $(\mathrm{n}(\%))$

$39(18.8)$

At least one hospitalisation in the past

12 months $(\mathrm{n}(\%))^{*}$

$142(68.3)$

Clinical status stable for the past

3 months (n (\%))

$184(86.0)$

NYHA FC I: II: III: IV (\%)

6-minute walk distance $(\mathrm{m})$

10.6: $48.1: 37.5: 3.9$

$\mathrm{PAH}$-specific treatments $(\mathrm{n}(\%))^{* *}$

$431 \pm 109(n=147)$

No treatment

$49(23.6)$

Monotherapy

$85(40.9)$

Double combination therapy

$66(31.7)$

Triple combination therapy

8 (3.9)

$\mathrm{FC}=$ functional class; $\mathrm{PAH}=$ pulmonary arterial hypertension; $\mathrm{PAHCHD}=$ pulmonary arterial hypertension associated with $\mathrm{CHD}$ Values are expressed as mean \pm standard deviation unless otherwise specified

*Hospitalisation due to PAH and/or CHD

**Endothelin receptor antagonist, phosphodiesterase type 5 inhibitor, and/or prostanoid 
The observed trend was a decrease in SF-36 scores with worsening NYHA functional class, except for the small group of patients in NYHA functional class IV $(n=8)$. The evolution of the health status compared with the previous year was more likely to be considered as favourable by patients with a worse NYHA functional class $(p=0.01)$.

Mean CAMPHOR symptom, functioning, and quality of life scores among CHD patients with pulmonary arterial hypertension were 8.8 (SD =5.6), $8.5(\mathrm{SD}=5.0)$, and 6.7 $(S D=5.6)$, respectively.

CAMPHOR scores were higher for female than for male patients ( $p=0.001$ for symptoms; $p=0.01$ for activity; $p=0.001$ for quality of life), but only the functioning score varied significantly with the phenotype of pulmonary arterial hypertension associated with CHD $(p=0.03$ and $p=0.04$ with and without gender adjustment, respectively, Table 2).

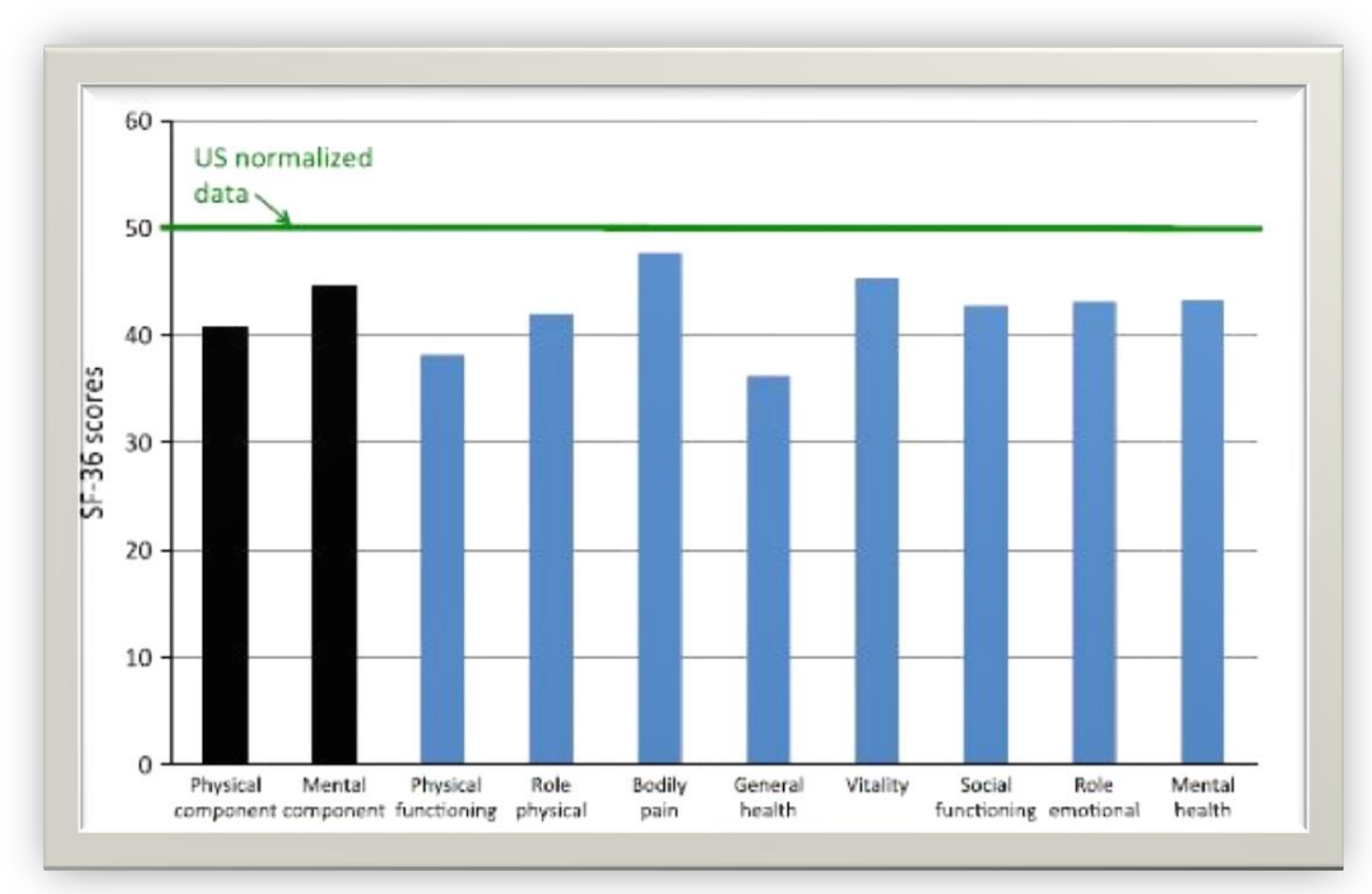

Figure 1. Quality of life impairment assessed by SF-36 in the adults adolescents CHD with pulmonary arterial hypertension quality of life assessment population compared with United States (US) normalised data $(n=208)$. Aggregate scores are shown in black and individual domains in blue. Higher scores indicate better quality of life. $p<0.0001$, except for bodily pain: $p=0.005$.

All CAMPHOR scores were significantly different between NYHA functional class with and without gender adjustment (Fig 3). The observed trend was an increase in impairment with worsening NYHA functional class. Correlations between the SF-36 and the CAMPHOR are shown in Table 3. All correlations were statistically significant in the anticipated direction. SF-36 summaries and CAMPHOR scores were strongly correlated, with the exception of the CAMPHOR functioning and SF-36 mental component summary, which presented a weaker correlation. 
Table 2. SF-36, Cambridge Pulmonary Hypertension Outcome Review (CAMPHOR), and Hospital Anxiety and Depression Scale (HADS) scores according to pulmonary arterial hypertension (PAH)-CHD phenotypes.

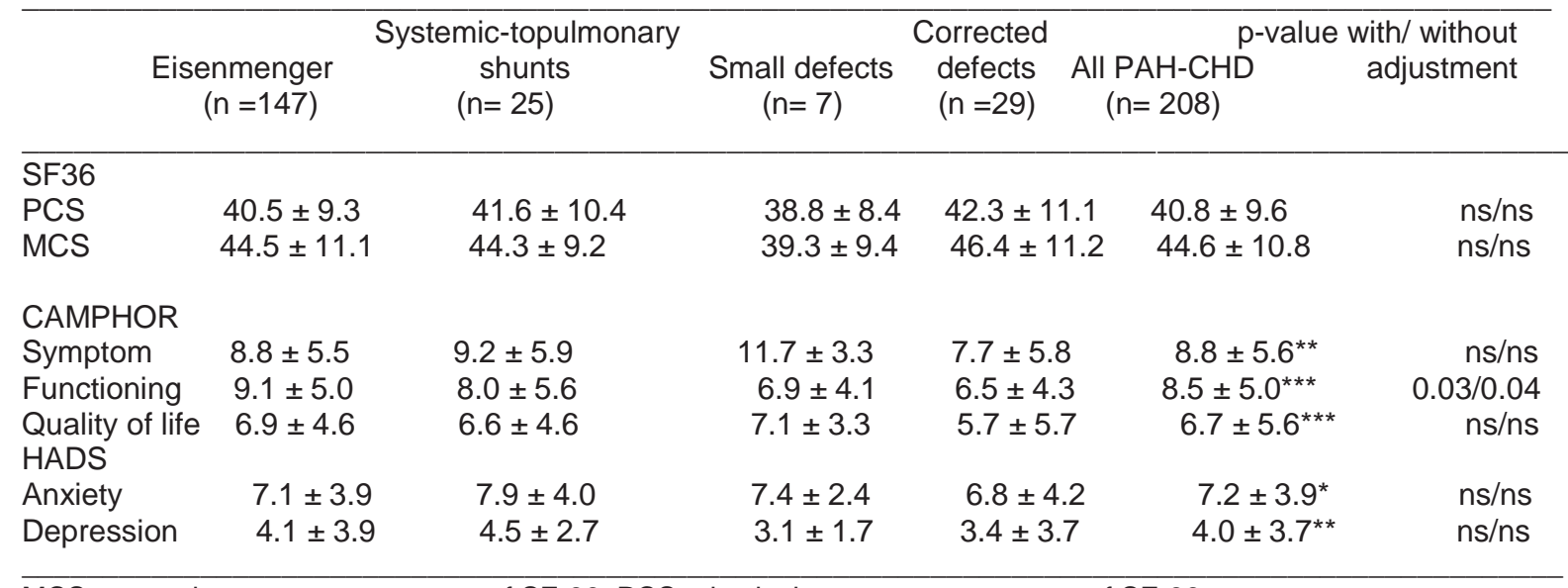

MCS= mental component summary of SF-36; PCS=physical component summary of SF-36

Values are expressed as mean \pm standard deviation

${ }^{*} \mathrm{n}=204$

** $\mathrm{n}=206$

$* * * n=207$

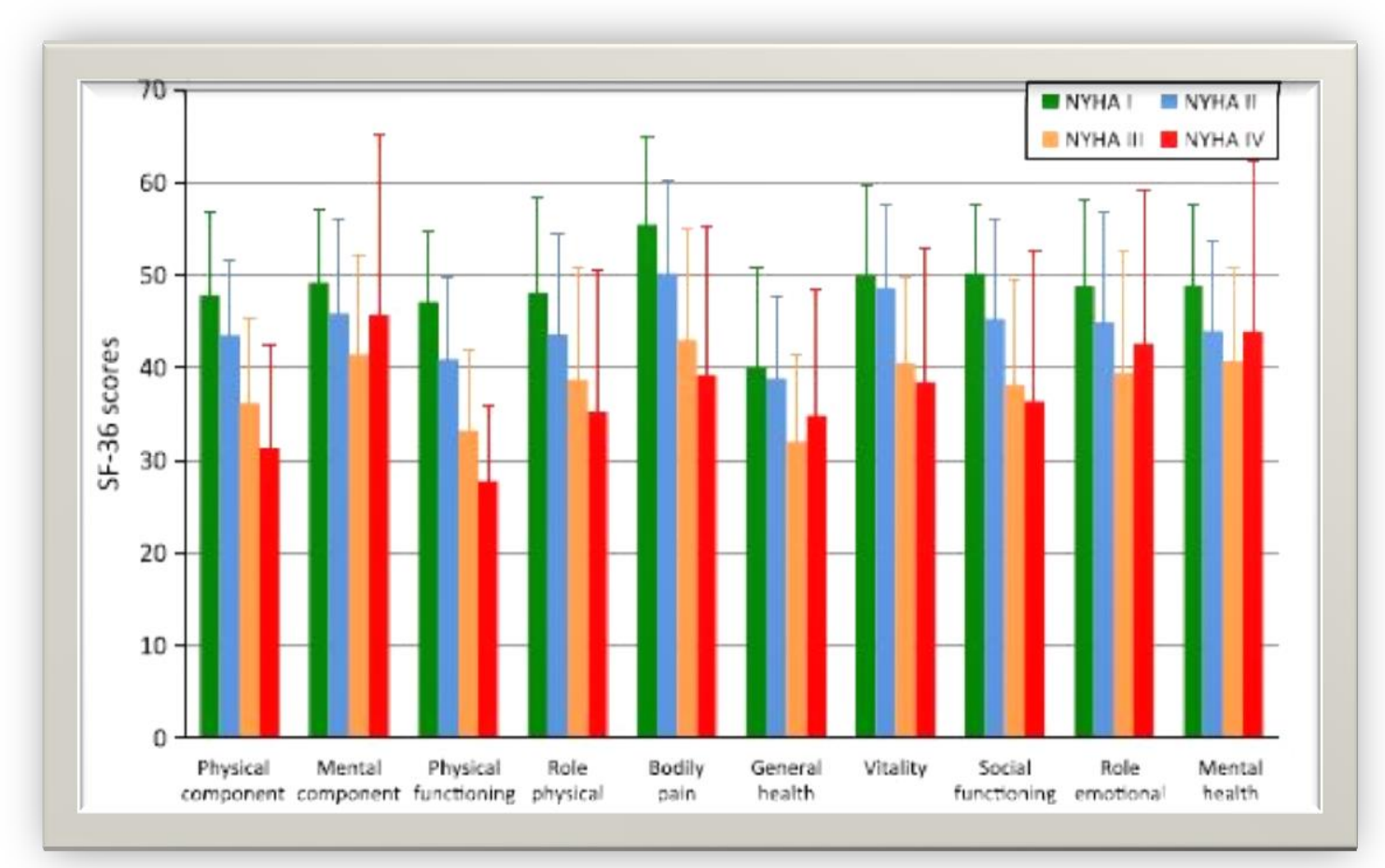

Figure 2. SF-36 scores (mean \pm standard deviation) for patients in NYHA functional classes I-IV $(n=$ 208). Higher scores indicate better quality of life. All SF-36 scores were significantly different between NYHA functional classes, with and without gender adjustment by analysis of variance. 


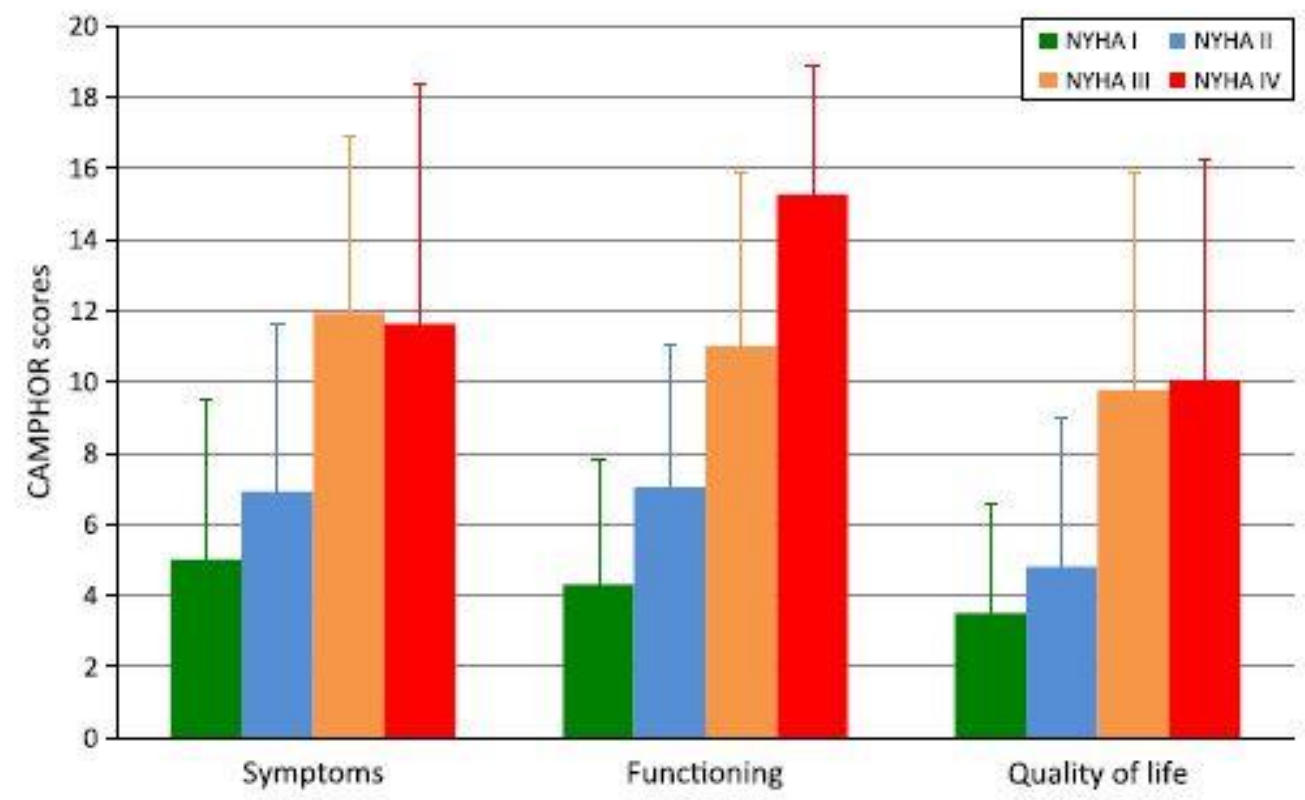

Figure 3. Cambridge Pulmonary Hypertension Outcome Review (CAMPHOR) scores (mean \pm standard deviation) for patients in NYHA functional classes I-IV $(n=208)$. Higher scores indicate higher impairment. All CAMPHOR scores were significantly different between NYHA functional classes, with and without gender adjustment by analysis of variance $(p<0.001)$.

\section{HADS questionnaire scores}

HADS scores for anxiety and depression were $7.2(\mathrm{SD}=3.9)$ and $4.0(\mathrm{SD}=3.7)$, respectively; they were $\geqslant 8$ for 64 patients (31.4\%) and 19 patients $(9.2 \%)$, respectively. HADS scores were higher for female than for male patients $(p=0.01$ and $p=0.001$, respectively), but did not vary significantly with the phenotype of pulmonary arterial hypertension associated with CHD (Table 2), even after gender adjustment. Scores were significantly different between NYHA functional class for both anxiety and depression with and without gender adjustment (Fig 4). The observed trend was an increase in HADS scores with worsening NYHA functional class, except for the small group of patients in NYHA functional class IV $(\mathrm{n}=8)$.

\section{Factors affecting SF-36 and CAMPHOR}

The results from univariate and multivariate regression analyses are presented in Table 4. Female gender was a factor affecting the SF-36 physical component summary and mental component summary as well as the CAMPHOR scores in univariate analyses, but significance was not maintained in the multivariate analyses, except for the SF-36 mental component summary.

Table 3. Correlations between SF-36 and Cambridge Pulmonary Hypertension Outcome Review (CAMPHOR).

\begin{tabular}{lcc}
\hline & SF-36 PCS & SF-36 MCS \\
\hline $\begin{array}{l}\text { CAMPHOR } \\
\text { CAmptom }\end{array}$ & $\mathrm{R}=-0.65, \mathrm{p}<0.0001$ & $\mathrm{R}=-0.59, \mathrm{p}<0.0001$ \\
$\begin{array}{l}\text { Functioning } \\
\text { CAMPHOR } \\
\text { quality of life }\end{array}$ & $\mathrm{R}=-0.69, \mathrm{p}<0.0001$ & $\mathrm{R}=-0.31, \mathrm{p}<0.0001$ \\
& $\mathrm{R}=-0.57, \mathrm{p}<0.0001$ & $\mathrm{R}=-0.56, \mathrm{p}<0.0001$ \\
\hline
\end{tabular}

$\overline{\mathrm{MCS}}=$ mental component summary; PCS=physical component summary 


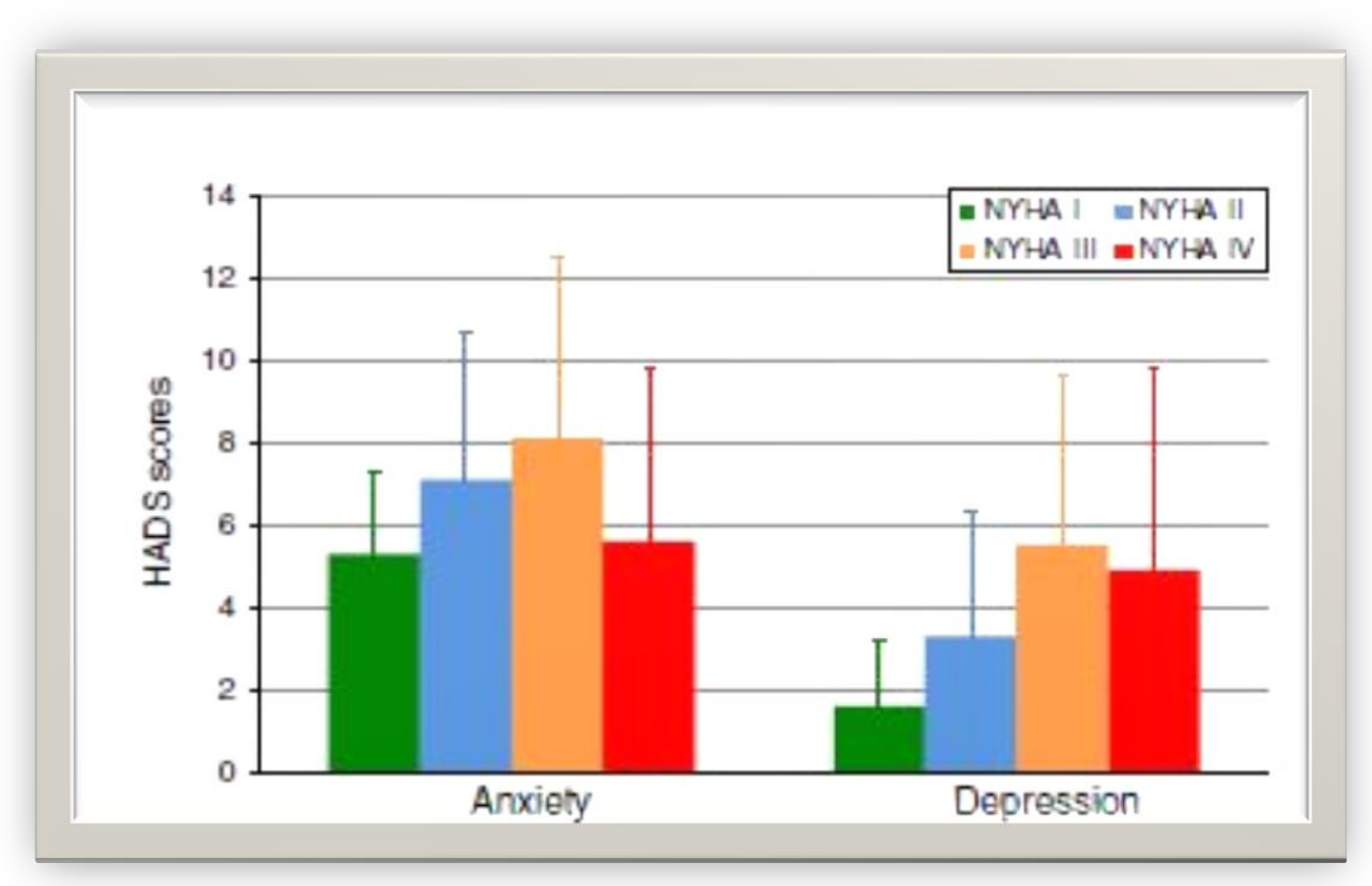

Figure 4. Hospital Anxiety and Depression Scale (HADS) scores (mean \pm standard deviation) for patients in NYHA functional classes I-IV $(n=191)$. Higher scores indicate higher anxiety/ depression. Scores were significantly different between NYHA functional classes for both anxiety and depression $(p=0.02$ and $p<0.0001$, respectively) with and without gender adjustment by analysis of variance.

Table 4. Impact of demographic and clinical factors on health-related quality of life.

p-values from (univariate) multivariate regression analyses

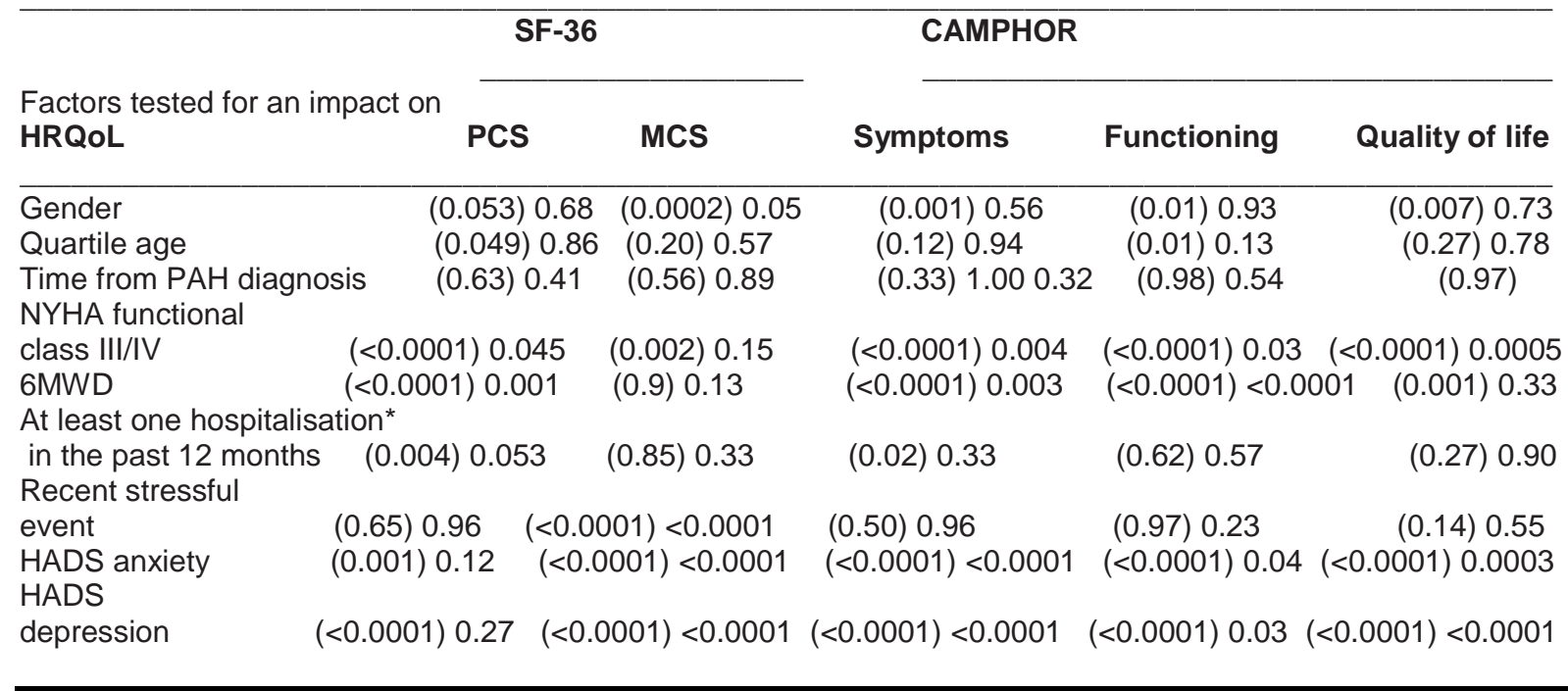

CAMPHOR=Cambridge Pulmonary Hypertension Outcome Review; HADS =Hospital Anxiety and Depression Scale; $\mathrm{HRQoL}=$ health-related quality of life; $\mathrm{MCS}=$ mental component summary; $\mathrm{PAH}=$ pulmonary arterial hypertension; $\mathrm{PCS}=$ physical component summary; $6 \mathrm{MWD}=6$-minute walk distance * Hospitalisation due to $\mathrm{PAH}$ and/or $\mathrm{CHD}$ 
Age only had an impact on the SF-36 physical component summary and CAMPHOR functioning in univariate analyses. The time from diagnosis of pulmonary arterial hypertension had no impact on health-related quality of life. In the multivariate analysis, NYHA functional class III/IV was related to all SF-36 and CAMPHOR scores, with the exception of the SF-36 mental component summary, whereas the 6-minute walk distance was associated with the SF-36 physical component summary and the CAMPHOR symptoms and functioning. HADS scores impacted all health-related quality of life scores in univariate analyses and the effect was confirmed in multivariate analyses except for the SF-36 physical component summary. At least one hospitalisation for pulmonary arterial hypertension and/or CHD during the past 12 months was a factor affecting the SF-36 physical component summary and the CAMPHOR symptoms only in univariate analyses, whereas a recent stressful event affected the SF-36 mental component summary in multivariate analyses.

\section{Discussion}

The ACHILLE study is a prospective, cross-sectional, multicentre, observational study, which investigated the health-related quality of life of a large cohort of208 CHD patients with pulmonary arterial hypertensionin 14 specialised CHD centres. Although the patients presented with a stable disease, they had an impaired health-related quality of life as assessedusing both generic and pulmonary hypertensionspecific questionnaires. NYHA functional class and HADS scores were predictive of most health-related quality of life scores.

Health-related quality of life has become a matter of increasing interest in a number of chronic diseases including pulmonary arterial hypertension,27-29 buta few studies have focussed on patients with pulmonary arterial hypertension associated with CHD. Only one study by Becker-Grunig et al 12 has described the health-related quality of life of 20 patients with pulmonary arterial hypertension associated with CHD. Other studies are small series involving patients with Eisenmenger syndrome.13-17

In our study, we included 208 patients presenting with all four clinical phenotypes of pulmonary arterial hypertension associated with CHD:18 Eisenmenger syndrome, pulmonary arterial hypertension associated with systemic-to-pulmonary shunts, small defects, and corrected defects. In these patients, health-related quality of life was impaired, particularly in the physical domains of the SF-36, which is consistent with the findings of the abovementioned small studies. Although health-related quality of life is compromised in our patients with pulmonary arterial hypertension associated with CHD, impairment appears less severe than in patients with other types of pulmonary arterial hypertension. 27-29 This might be due to the fact that CHD patients with pulmonary arterial hypertension have lived with CHD since childhood and may have adapted to their illness.30 Besides, some patients might be aware of a better prognosis of their disease's subgroup, and this might influence the way they estimate their quality of life, especially in the mental dimensions.

Patients with pulmonary arterial hypertension associated with CHD are a heterogeneous population, and the four clinical phenotypes have different clinical, functional, and haemodynamic characteristics as well as diverse patterns of survival. ${ }_{11}$ We compared the health-related quality of life and level of anxiety/depression of patients across the four phenotypes, but no significant difference was observed except for the functioning dimension of the CAMPHOR. Functional disability was increased in patients with 
Eisenmenger syndrome in line with the comparative study of Manes et al,31 which reported the worst functional capacity and haemodynamics for these patients. The lack of significant difference of other health-related quality of life and anxiety/ depression parameters across subgroups of pulmonary due to the small numbers of patients in some of the subgroups, but it cannot be excluded that pulmonary arterial hypertension is predominantly impairing health-related quality of life, regardless of the associated cardiac defect. This is substantiated by a study by Muller et al 15 who reported that health-related quality of life is more impaired in pulmonary arterial hypertension due to Eisenmenger syndrome associated with a simple cardiac defect than in complex cyanotic CHD with pulmonary stenosis.

In our study, NYHA functional class was an independent predictor for all domains of the CAMPHOR and for the physical component summary of the SF-36, whereas the 6minute walk distance was only predictive of the symptoms and functioning domains of the CAMPHOR. Such associations between health-related quality of life and measures of the functional status - NYHA functional class and to a lesser degree the 6-minute walk distance - have been demonstrated consistently in patients with pulmonary arterial hypertension.9,27,29

Owing to diminished health-related quality of life, decreased exercise capacity, financial burden of treatment, unemployment, and an uncertain prognosis, patients with pulmonary arterial hypertension associated with CHD may be more vulnerable to developing depression and anxiety.8 The high prevalence of depression and anxiety in patients with pulmonary arterial hypertension has been known for many years.32-35 In the patients of our study, the HADS anxiety scores were comparable with those reported for patients with various types of pulmonary arterial hypertension, 32,35 whereas depression scores were indicative of a less depressed population. As reported in other studies, 36 we found that mental disorders were significantly associated with health-related quality of life, suggesting that mental disorders should be considered in the management of these patients.

Health-related quality of life is patients' own expectations and perceptions of how their health affects them. It is therefore subjective and time dependent.

Adjustment to pulmonary arterial hypertension has been reported in other studies, 37 but we did not observe an effect of time since diagnosis on patients' health-related quality of life. Nonetheless, an interesting observation was that patients in NYHA functional class IV tended to perform better in some of the mental domains of the SF-36 and in the HADS than patients in NYHA functional class III. These results could suggest a phenomenon of psychological resilience in patients suffering from an incurable disease at an advanced stage, although they should be interpreted cautiously, given the limited number of patients in NYHA functional class IV.

Measurements of health-related quality of life in patients with pulmonary arterial hypertension have been reported in a number of epidemiological studies27-29 and clinical trials 38 but they are often overlooked in routine clinical practice, possibly because they are still perceived as subjective, cumbersome, and unreliable. Yet, health-related quality of life questionnaires provide valuable information on the burden of pulmonary arterial hypertension on the patient's day-to-day living and have also been associated with prognosis.39,40 In our study, the generic SF-36 and pulmonary hypertensionspecific CAMPHOR questionnaires presented strong inter-domain correlations as observed 
previously in patients with pulmonary hypertension 41 and were strongly associated with therapeutic objectives as assessed by NYHA functional class and the 6-minute walk distance, supporting their use in clinical practice.

The quality of life assessment of our patients is certainly subjective, but not less essential. Today, few congenital cardiologists evaluate in regular follow-up the actual quality of life of their patients. As in many other chronic diseases, measurement of patient-related outcomes has been given more or more attention in congenital cardiology, but mostly when carrying out clinical research trials. 42 In their routine practice, physicians mostly rely on clinical symptoms and disease complications but usually have a wrong estimation of their patients' overall life satisfaction 43,44. Yet, approaching the physical and psychosocial aspects of quality of life can enlarge the doctor-patient interaction and help decision making in routine clinical practice.

Therefore, we recommend for pulmonary hypertension CHD specialists to regularly assess the health-related quality of life of their patients - for instance, during the annual or semi-annual medical check-up. A specialist nurse could probably be dedicated to this assessment. As similar results were obtained in our study by both generic and specific questionnaires, we recommend using the SF-36 for clinical routine health-related quality of life assessment. This generic questionnaire is rapidly filled-out, easily scored, and also enables comparison with the healthy population in many countries. Moreover, symptoms of anxiety and/or depression should be systematically evaluated in routine clinical practice. The HADS questionnaire is a simple tool for pulmonary hypertension CHD specialists, but in routine follow-up patients should be evaluated and potentially regularly followed-up by a psychologist or a psychiatrist according to individual needs.

\section{Limitations}

This cross-sectional, multicentre, observational study has a number of inherent limitations as it was designed to describe the health-related quality of life of patients with pulmonary arterial hypertension associated with CHD with no investigation of the evolution of this health-related quality of life with time. The patient population is limited to the more severe patients followed-up in selected centres with expertise in pulmonary vascular medicine. Patients with cognitive limitations who could not fill the questionnaire were excluded, and are therefore not represented in our group of patients with pulmonary arterial hypertension associated with CHD. At inclusion, a high proportion of patients (23.6\%) did not receive any pulmonary arterial hypertension-specific therapies, which could have had an impact on health-related quality of life. The study enrolled a large number of patients with pulmonary arterial hypertension associated with CHD, but some subgroups were small, such as the subgroup of patients in NYHA functional class IV $(n=8)$ and patients with small defects $(n=7)$, which precluded some statistical analyses and interpretation. Patients' awareness of prognosis might have influenced their selfreported health-related quality of life scores and could represent a possible confounding factor, especially when comparing the different subgroups where prognosis is known to be different; however, data comparing these different prognoses were published after patients were enrolled in the ACHILLE study, suggesting limited bias. 31

\section{Conclusion}

This study shows in a large patient population that CHD with pulmonary arterial hypertension is associated with impaired health-related quality of life, as assessed using both the generic SF-36 and the pulmonary hypertension-specific CAMPHOR 
questionnaires. Confronted with numerous life stressors, these patients are also prone to anxiety and depression. Health-related quality of life, anxiety, and depression should be more systematically evaluated in routine clinical practice, as tools have demonstrated validity and reliability and psychological support and/or treatment may be beneficial for the patient.

\section{Acknowledgements}

The authors thank the patients who kindly participated in the study. The authors also thank the investigators of the ACHILLE study: L. Iserin, M. Ladouceur, O. Sanchez, A. Legendre: M3C-Georges Pompidou European Hospital, Paris; O. Sitbon: Bicêtre University Hospital, Le Kremlin-Bicêtre; L. Le Gloan: Guillaume and René Laennec Hospital, Nantes; A. Sénéchal, V. Cottin, A. Soufi, J. Traclet, C. Khouatra: Louis Pradel Hospital, cardiology and respiratory diseases departments- Bron; F. Lalombarda, E. Bergot, P. Maragnes: Côte de Nacre Hospital, Caen; E. Barre: CharlesNicolle Hospital, Rouen; J.-M. Schleich: University Hospital, Rennes; C. Dauphin, J.-R. Lusson: GabrielMontpied University Hospital, Clermont-Ferrand; M. Levy: M3C-Necker University Hospital, Paris; X. Iriart, M. de Guillebon: Haut-Lévêque University Hospital, Bordeaux; H. Bouvaist: Albert Michallon Hospital, La Tronche; A. Baruteau, V. Lambert, M. Gouton: Marie Lannelongue Chirurgical centre, Le Plessis Robinson. The authors also thank $L$. Iserin (M3C-Georges Pompidou European Hospital, Paris, France) for her comments on the final manuscript. Medical writing assistance was provided by S. Ertel (Sundgau Medical Writers, Habsheim, France), funded by Actelion Pharmaceuticals Ltd. Financial

\section{Support}

This work was supported by Actelion Pharmaceuticals France. Actelion Pharmaceuticals France provided funding for logistical support, project management, data management, and statistical analyses.

\section{Conflicts of Interest}

P.A. reports personal fees and non-financial support from Actelion Pharmaceuticals France, non-financial support from Bioprojet, Bayer, Novartis, Servier, and Roche. A.B. and P.C. declare no conflicts of interest. V.G. is a full-time employee of Actelion Pharmaceuticals France as medical director. X.J. reports personal fees from Actelion Pharmaceuticals France, GSK, and Pfizer. J.B.T., S.C., and D.B. report personal fees from Actelion Pharmaceuticals France. P.G. reports personal fees from Actelion Pharmaceuticals France and Abbott. P.G. reports personal fees from Actelion Pharmaceuticals France and Abbott. Ethical Standards The authors assert that all procedures contributing to this work comply with the ethical standards of the relevant national guidelines on human experimentation and with the Helsinki Declaration of 1975, revised in 2008, and has been approved by the CCTIRS (national advisory committee for data processing in health research) on 18 April, 2012 (registration number 12.101).

\section{References}

1. Hoffman JI, Kaplan S. The incidence of congenital heart disease. J Am Coll Cardiol 2002; 39: 1890-1900.

2. Marelli AJ, Mackie AS, lonescu-lttu R, Rahme E, Pilote L. Congenital heart disease in the general population: changing prevalence and age distribution. Circulation 2007; 115: 163-172.

3. Khairy P, Ionescu-Ittu R, Mackie AS, Abrahamowicz M, Pilote L, Marelli AJ. Changing mortality in congenital heart disease. J Am Coll Cardiol 2010; 56: 1149-1157.

4. Duffels MG, Engelfriet PM, Berger RM, et al. Pulmonary arterial hypertension in congenital heart disease: an epidemiologic perspective from a Dutch registry. Int J Cardiol 2007; 120: 198-204.

5. Beghetti M, Tissot C. Pulmonary arterial hypertension in congenital heart diseases. Semin Respir Crit Care Med 2009; 30: 421-428. 
6. Lowe BS, Therrien J, Ionescu-Ittu R, Pilote L, Martucci G, Marelli AJ. Diagnosis of pulmonary hypertension in the congenital heart disease adult population impact on outcomes. J Am Coll Cardiol 2011; 58: 538-546.

7. Dimopoulos K, Inuzuka R, Goletto S, et al. Improved survival among patients with Eisenmenger syndrome receiving advanced therapy for pulmonary arterial hypertension. Circulation 2010; 121: 20-25.

8 Guillevin L, Armstrong I, Aldrighetti R, et al. Understanding the impact of pulmonary arterial hypertension on patients' and carers' lives. Eur Respir Rev 2013; 22: 535-542.

9. Matura LA, McDonough A, Carroll DL. Health-related quality of life and psychological states in patients with pulmonary arterial hypertension. J Cardiovasc Nurs 2014; 29: 178-184.

10. Chen H, Taichman DB, Doyle RL. Health-related quality of life and patient-reported outcomes in pulmonary arterial hypertension. Proc Am Thorac Soc 2008; 5: 623-630.

11. McLaughlin VV, Archer SL, Badesch DB, et al. ACCF/AHA 2009 expert consensus document on pulmonary hypertension a report of the American College of Cardiology Foundation Task Force on Expert Consensus Documents and the American Heart Association developed in collaboration with the American College of Chest

Physicians; American Thoracic Society, Inc.; and the Pulmonary Hypertension Association. J AmColl Cardiol 2009; 53: 1573-1619.

12. Becker-Grunig T, Klose H, Ehlken N, et al. Efficacy of exercise training in pulmonary arterial hypertension associated with congenital heart disease. Int J Cardiol 2013; 168: 375-381.

13. Duffels MG, Vis JC, van Loon RL, et al. Down patients with Eisenmenger syndrome: is bosentan treatment an option? I J Cardiol 2009; 134: 378-383.

14. Duffels MG, Vis JC, van Loon RL, et al. Effect of bosentan on exercise capacity and quality of life in adults with pulmonary arterial hypertension associated with congenital heart disease with and without Down's syndrome. Am $J$ Cardiol 2009; 103: 1309-1315.

15. Muller J, Hess J, Hager A. Exercise performance and quality of life is more impaired in Eisenmenger syndrome than in complex cyanotic congenital heart disease with pulmonary stenosis. Int J Cardiol 2011; 150: 177-181.

16. Tay EL, Papaphylactou M, Diller GP, et al. Quality of life and functional capacity can be improved in patients with Eisenmenger syndrome with oral sildenafil therapy. Int J Cardiol 2011; 149: 372-376.

17. Tay EL, Peset A, Papaphylactou M, et al. Replacement therapy for iron deficiency improves exercise capacity and quality of life in patients with cyanotic congenital heart disease and/or the Eisenmenger syndrome. Int J Cardiol 2011; 151: 307-312.

18. Simonneau G, Robbins IM, Beghetti M, et al. Updated clinical classification of pulmonary hypertension. J Am Coll Cardiol 2009; 54: S43-S54.

19. Ware JE Jr, Sherbourne CD. The MOS 36-item short-form health survey (SF-36). I. Conceptual framework and item selection. Med Care 1992; 30: 473-483.

20. Ware JE Jr, Gandek B, Kosinski M, et al. The equivalence of SF-36 summary health scores estimated using standard and countryspecific algorithms in 10 countries: results from the IQOLA Project. International Quality of Life Assessment. J Clin Epidemiol 1998; 51: 1167-1170.

21. Perneger TV, Leplege A, Etter JF, Rougemont A. Validation of a French-language version of the MOS 36-Item Short Form Health Survey (SF-36) in young healthy adults. J Clin Epidemiol 1995; 48: 1051-1060.

22. Perneger TV, Leplege A, Etter JF. Cross-cultural adaptation of a psychometric instrument: two methods compared. J Clin Epidemiol 1999; 52: 1037-1046.

23. McKenna SP, Doughty N, Meads DM, Doward LC, Pepke-Zaba J. The Cambridge Pulmonary Hypertension Outcome Review (CAMPHOR): a measure of health-related quality of life and quality of life for patients with pulmonary hypertension. Qual Life Res 2006; 15: 103-115.

24. Coffin D, Duval K, Martel S, et al. Adaptation of the Cambridge PulmonaryHypertension Outcome Review (CAMPHOR) into French- Canadian and English-Canadian. Can Respir J 2008; 15: 77-83.

25. Zigmond AS, Snaith RP. The hospital anxiety and depression scale. Acta Psychiatr Scand 1983; 67: $361-370$.

26. Crawford JR, Henry JD, Crombie C, Taylor EP. Normative data for the HADS from a large non-clinical sample. Br J Clin Psychol 2001; 40: 429-434.

27. Taichman DB, Shin J, Hud L, et al. Health-related quality of life in patients with pulmonary arterial hypertension. Respir Res 2005; 6: 92.

28. Zlupko M, Harhay MO, Gallop R, et al. Evaluation of disease-specific health-related quality of life in patients with pulmonary arterial hypertension. Respir Med 2008; 102: 1431-1438.

29. Chen H, De Marco T, Kobashigawa EA, Katz PP, Chang VW, Blanc PD. Comparison of cardiac and pulmonaryspecific quality of life measures in pulmonary arterial hypertension. Eur Respir J 2011; 38: 608-616.

30. Dimopoulos K, Giannakoulas G, Wort SJ, Gatzoulis MA. Pulmonary arterial hypertension in adults with congenital heart disease: distinct differences from other causes of pulmonary arterial hypertension and management implications. Curr Opin Cardiol 2008; 23: 545-554.

31. Manes A, Palazzini M, Leci E, Bacchi Reggiani ML, Branzi A, Galie N. Current era survival of patients with pulmonary arterial hypertension associated with congenital heart disease: a comparison between clinical subgroups. Eur Heart J 2014; 35: 716-724.

32. Shafazand S, Goldstein MK, Doyle RL, Hlatky MA, Gould MK. Health-related quality of life in patients with pulmonary arterial hypertension. Chest 2004; 126: 1452-1459.

33. White J, Hopkins RO, Glissmeyer EW, Kitterman N, Elliott CG. Cognitive, emotional, and quality of life outcomes in patients with pulmonary arterial hypertension. Respir Res 2006; 7: 55.

34. McCollister DH, Beutz M, McLaughlin V, et al. Depressive symptoms in pulmonary arterial hypertension: prevalence and association with functional status. Psychosomatics 2010; 51: 339-339 e8.

35. Yorke J, Armstrong I. The assessment of breathlessness in pulmonary arterial hypertension: reliability and validity of the Dyspnoea-12. Eur J Cardiovasc Nurs 2013.

36. Halank M, Einsle F, Lehman S, et al. Exercise capacity affects quality of life in patients with pulmonary hypertension. Lung 2013; 191: 337-343. 
37. McDonough A, Matura LA, Carroll DL. Symptom experience of pulmonary arterial hypertension patients. Clin Nurs Res 2011; 20: 120-134.

38. Rival G, Lacasse Y, Martin S, Bonnet S, Provencher S. Effect of pulmonary arterial hypertension-specific therapies on healthrelated quality of life: a systematic review. Chest 2014; 146: 686-708.

39. Fernandes CJ, Martins BC, Jardim CV, et al. Quality of life as a prognostic marker in pulmonary arterial hypertension. Health Qual Life Outcomes 2014; $12: 130$.

40. Blok IM, van Riel AC, Schuuring MJ, et al. Decrease in quality of life predicts mortality in adult patients with pulmonary arterial hypertension due to congenital heart disease. Neth Heart J 2015; 23: 278-284.

41. Gomberg-Maitland M, Thenappan T, Rizvi K, Chandra S, Meads DM, McKenna SP. United States validation of the Cambridge Pulmonary Hypertension Outcome Review (CAMPHOR). J Heart Lung Transplant 2008; 27: 124-130.

42. Moons P. Patient-reported outcomes in congenital cardiac disease: are they as good as you think they are? Cardiol Young 2010; 20 (Suppl 3): 143-148.

43. Moons P, Budts W, De Geest S. Critique on the conceptualization of quality of life: a review and evaluation of different conceptual approaches. Int J Nurs Stud 2006; 43: 891-901.

44. Marino BS, Tomlinson RS, Drotar D, et al. Quality-of-life concerns differ among patients, parents, and medical providers in children and adolescents with congenital and acquired heart disease. Pediatrics 2009; 123: e708-e715. 